\title{
Robust Uplink Resource Allocation in CDMA Cellular Radio Systems
}

\author{
Erik Geijer Lundin, Fredrik Gunnarsson and Fredrik Gustafsson \\ Division of Automatic Control \\ Department of Electrical Engineering, Linköping University \\ SE-581 83 LINKÖPING, SWEDEN \\ \{geijer, fred, fredrik\}@ isy.liu.se*
}

\begin{abstract}
Radio resource management (RRM) in cellular radio system is an example of automatic control where there, one way or another, is limited information. Either the control algorithm is hosted in a central node and then receives limited information from a wide area. Or, the algorithm is located in a local node (base station) and then has only knowledge of the situation in the corresponding cell. Despite these limitations, good utilization of available resources is required without jeopardizing system stability.

The proposed RRM algorithm applies to the uplink (mobile to base station) of a code division multiple access (CDMA) cellular system and uses decisions made both in a central node and in the base stations. The central node, operating on a slow update rate, guarantees system stability while the base stations can make fast decisions to improve performance based on rapid changes in the local radio environment.

Simulations indicate that using the proposed combination of centralized and decentralized control better resource utilization given an upper limit on the received interference power.Moreover, as opposed to using decentralized control, the algorithm guarantees system stability.
\end{abstract}

\section{INTRODUCTION}

The control aspect of radio resource management may be stated as: provide as high quality of service as possible by assigning users radio resources without jeopardizing system stability, despite user movement and external disturbance. An overview of radio resource management is given in [1]

The purpose of all RRM algorithms is to set the target values for the power control algorithm of each uplink connection such that the system is feasible, while providing as high quality of service as possible. A system is feasible if it exists finite transmission powers such that all users perceive an acceptable quality of the allocated service (typically related to the uplink signal to noise ratio) [2].

System feasibility is a system property, and as such depends on the situation in the entire system as a whole. If each base station distributes resources to the users without respect to the situation in surrounding cells in a multi cell system, system feasibility is thus obviously not guaranteed.

To efficiently solve the RRM problem it is therefore our strong belief that the control algorithm should be a combination of centralized and decentralized control. Centralization is required to guarantee system feasibility. Because of the

${ }^{*}$ This work is supported by the Swedish Agency for Innovation Systems (VINNOVA), Information Systems for Industrial Control and Supervision (ISIS) and in cooperation with Ericsson Research, which are all acknowledged. limited bandwidth between local nodes and the central node, the detailed information can not be made available in the central node. Instead, course information and/or sparsely sampled data is sent to the central node. Decentralization gives additional performance gains by using detailed information on the immediate radio environment and current user demands. The information flow can be visualized as in Figure 1, where the central node controls the resources using control signals $s_{j}, j=1,2, \ldots, B$ such that system stability is guaranteed while the local nodes perform the actual resource assignment to the separate users. In this context, resource (re-)assignment means that each base station $j$ allocates resources $\gamma_{i}$ to all served users $i \in c_{j}$, where $c_{j}$ is the set of users served by base station $j$.

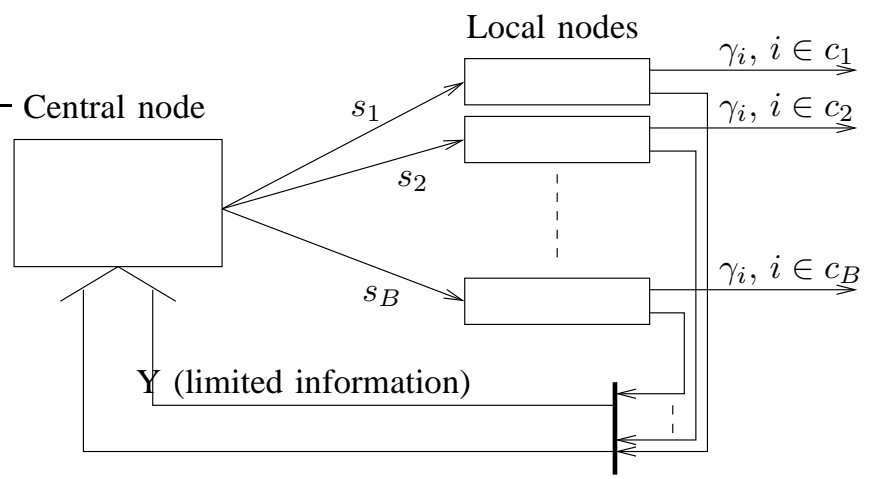

Fig. 1. Information flow in a decentralized RRM algorithm.

An addition to WCDMA (the radio interface of UMTS) called Enhanced Uplink is currently being standardized by 3GPP [3]. The objective is to enable high uplink data rates and short delays. This will be done by decentralizing most of the resource control to the base stations and using more efficient retransmissionprotocol [4].

The resource allocation problem can, like many control problems, be formulated as an optimization problem.

$$
\begin{gathered}
\max _{\gamma} U(\gamma) \\
\text { s.t. system is feasible, }
\end{gathered}
$$

where $\gamma$ is the vector of users' signal-to-noise ratios. The utility function, $U(\gamma)$, can be seen as a realization of the quality of service demands. It should therefore reflect the user satisfaction. Speech users and data users have different 
demands, and should therefore use different utility functions [5].

Since the limiting resource in the uplink of a CDMA cellular system is the received interference power, a resource control algorithm should consider this quantity. The difference in performance between algorithms studying the introduced interference power and those simply counting the number of users in each cell has been established in many contributions [6], [7].

Examples where an optimization problem has been defined and solved in a single cell system are [8], [9]. Examples of where all base stations of a multi cell system have been given a common rule and no centralized node is used are [10], [11], [12]. A drawback with the methods given in the above references is that the base stations require knowledge of the current received interference power, which is generally considered hard to measure accurately.

We propose an algorithm consisting of two levels of optimization; in the central node as well as in each base station. In short, the central node receives limited power gain information from each base station, $Y$. From this, it decides how much resources $\left(s_{k}\right)$ each base station can assign to its users $\left(\gamma_{i}\right)$. The base stations then assign resources to the users. This, together with an agreement between the central node and the base stations, guarantees feasibility. The information flow of the algorithm can be visualized as in Figure 1.

An advantage with our approach is that the base station controls the resources and can therefore assign a user with greater needs more resources on the behalf of other users. This is useful when the radio traffic is burst, or when users have different priorities. Furthermore, we only use information readily available in a practical cellular system.

Section II explains the notation and system model used. After that, a criteria for system feasibility is provided in Section III. The radio resource management algorithms, which assign resources to the users, studied in this contribution are explained in Section IV. Simulations were used to establish the performance of the algorithms. Results of those are discussed in Section $\mathrm{V}$ before conclusions are made in Section VI.

\section{System ModeL}

Consider a scenario consisting of $B$ base stations and $M$ users. User $i$ is solely connected to base station $K_{i}$ and the set of users connected to base station $j$ is denoted $c_{j}$. The power gain between user $i$ and base station $j$ is denoted $g_{i, j}<1$. We will also use relative power gain between user $i$ and base station $j, z_{i, j} \triangleq \frac{g_{i, j}}{g_{i, K_{i}}}$.

Each user $i$ is transmitting with power $p_{i}$. The uplink channel is modeled by the power gain $g_{i, K_{i}}$. The bit rate and bit error probability perceived by user $i$ is related to the carrier-to-total-interference-ratio (CTIR) ${ }^{1}$,

$$
\beta_{i}=\frac{p_{i} g_{i, K_{i}}}{I_{K_{i}}^{t o t}}
$$

The quantity $I_{j}^{t o t}$ is the total interference power in base station $j$. Power control adjusts the users' transmission powers, $p_{i}, i=1,2, \ldots, M$ to meet user individual target CTIR values, $\beta_{i}^{t g t}$, while mitigating time-varying disturbances.

Definition 1 (System Feasibility): Given all users' power gain to all base stations, $g_{i, j}, i \in\{1, M\}, j \in\{1, B\}$, and user individual target CTIR values, $\beta_{i}^{\text {tgt }}$, a system is feasible if it exists user individual finite positive transmission powers such that

$$
\beta_{i} \geq \beta_{i}^{t g t} \forall i
$$

Otherwise, the system is infeasible.

System feasibility is one out of many names used for this system property [2], [13]. The resource management can therefore be seen as mechanisms to assign CTIR levels to users, while ensuring system feasibility.

The primary resource of the uplink of a CDMA cellular system is the total received interference power, $I_{j}^{t o t}$ in base station $j$. This quantity is modeled as the sum of a base station specific background noise power, $N_{j}$, and contributions from all users in the entire network, $p_{i} g_{i, j}$,

$$
I_{j}^{t o t}=N_{j}+\sum_{i=1}^{M} p_{i} g_{i, j} .
$$

The notation is summarized in Table I.

TABLE I

SUMMARY OF THE INTRODUCED NOTATION.

\begin{tabular}{|c|c|}
\hline$M$ & Number of users \\
\hline$B$ & Number of base stations \\
\hline$p_{i}$ & Transmission power, user $i$ \\
\hline$g_{i, j}$ & Power gain between user $i$ and base station $j$ \\
\hline$z_{i, j}=\frac{g_{i, j}}{g_{i, K_{i}}}$ & Relative power gain between user $i$ and base station $j$ \\
\hline$K_{i}$ & Base station user $i$ is connected to \\
\hline$c_{j}$ & Set of users connected to base station $j$ \\
\hline$N_{j}$ & Total interference power in base station $j$ \\
\hline$I_{j}^{t o t}$ & Target CTIR, user $i$ \\
\hline$\beta_{i}^{t g t}$ &
\end{tabular}

\section{System FEASibiLity}

This section is devoted to finding a criteria for establishing whether or not the system is feasible, i.e., whether it exists finite transmission powers to support the users' target CTIR values.

If the resource control algorithm manages to maintain a reasonable load, it is fair to approximate the actual experienced CTIR, $\beta_{i}$, with $\beta_{i}^{t g t}$, i.e., power control manages to track $\beta_{i}^{t g t}$ despite time-varying disturbances $\left(g_{i, K_{i}}\right.$, interference from other connections etc.). We thus concentrate on

\footnotetext{
${ }^{1}$ The CTIR, $\beta$, is related to the more known carrier-to-interference ratio, $\gamma$ through $\beta=\frac{\gamma}{1+\gamma}$.
} 
the equations as if the power control has reached a steady state. User $i$ 's transmission power is then

$$
\beta_{i}^{t g t}=\frac{p_{i} g_{i, K_{i}}}{I_{K_{i}}^{t o t}} \Leftrightarrow p_{i}=\beta_{i}^{t g t} \frac{I_{K_{i}}^{t o t}}{g_{i, K_{i}}}
$$

The interference power contribution to base station $j$ from users connected to base station $k$ is

$$
I_{k, j} \triangleq \sum_{i \in c_{k}} p_{i} g_{i, j}=\sum_{i \in c_{k}} \beta_{i}^{t g t} \frac{g_{i, j}}{g_{i, K_{i}}} I_{K_{i}}^{t o t}=\sum_{i \in c_{k}} \beta_{i}^{t g t} z_{i, j} I_{k}^{t o t} .
$$

since $K_{i}$ for all users in $c_{k}$ is $k$. Let the element on row $k$ and column $j$ of the cross-coupling matrix $L$ be defined by

$$
L_{k, j} \triangleq \frac{I_{k, j}}{I_{k}^{t o t}}=\sum_{i \in c_{k}} \beta_{i}^{t g t} z_{i, j}
$$

Adding all cells' contributions to the background noise yields

$$
\begin{gathered}
I_{j}^{t o t}=N_{j}+\sum_{k=1}^{B} I_{k, j}= \\
N_{j}+\sum_{k=1}^{B} L_{k, j} I_{k}^{t o t}, j=1,2, \ldots, B .
\end{gathered}
$$

A compact expression for all the interference powers all base stations is

$$
I^{t o t}=N+L^{T} I^{t o t}
$$

where $I^{t o t}=\left[I_{j}^{t o t}\right]$.

Before providing a criteria for system feasibility, we introduce some additional notation. The eigenvalues of a general matrix $A$ will be denoted by $\lambda(A)$ and the maximum eigenvalue by $\bar{\lambda}(A)$, i.e.,

$$
\begin{array}{r}
\lambda(A) \triangleq \operatorname{eig}(A) \\
\bar{\lambda}(A) \triangleq \max \lambda(A) .
\end{array}
$$

Lemma 1: All elements of the vector $I^{t o t}=N+L^{T} I^{t o t}$ are positive and finite if $\bar{\lambda}(L)<1, L>0$ and $N>0$.

Proof: Let $B(\alpha)$ be the adjoint matrix of the characteristic matrix $\alpha E-A$, i.e., $B(\alpha) \triangleq(\alpha E-A)^{-1} \Delta(\alpha)$, where $\Delta(\alpha)$ is the characteristic polynomial of $A, \operatorname{det}(\alpha E-A)$ and $E$ is the identity matrix. If all elements of the matrix $L$ are positive $(L>0)$, Perron-Frobenius theory states that all elements of the matrix $B(\alpha)$ is non-negative and finite if $\bar{\lambda}(A)<\alpha$ [14]. By choosing $\alpha$ to 1 and $A$ to $L^{T}$, we get that if $\bar{\lambda}\left(L^{T}\right)<1$ then

$$
0<I^{t o t}=\left(E-L^{T}\right)^{-1} N<\infty,
$$

since all elements in the inverse as well as in $N$ are positive and finite. Using $\lambda(L)=\lambda\left(L^{T}\right)$ completes the proof.

Theorem 1: A wireless network with user's uplink CTIR targets, $\beta_{i}^{t g t}$, and uplink relative power gain $z_{i, j}$ is uplink feasible if $\bar{\lambda}(L)<1$. The resulting interference vector $I^{\text {tot }}$ is given by

$$
I^{t o t}=\left(E-L^{T}\right)^{-1} N
$$

Proof: There are finite transmission powers to support all users' target CTIR values, i.e., the system is feasible, if and only if all elements of a solution to Equation (3),
$I^{t o t}$, are positive. According to Lemma 1, all elements are positive if $\bar{\lambda}(L)<1$. The corresponding interference vector is the solution to Equation (3),

$$
I^{t o t}=N+L^{T} I^{t o t} \Leftrightarrow I^{t o t}=\left(E-L^{T}\right)^{-1} N .
$$

\section{Resource Management Algorithms}

\section{A. Centralized Algorithm}

Provided that the central node has knowledge of the entire power gain matrix $\left[g_{i, j}\right]$, it can make an optimal user target CTIR assignment, maximizing the chosen utility function. According to Theorem 1, the system is feasible if $\bar{\lambda}(L)<1$. An example of an optimization problem is thus ${ }^{2}$

$$
\begin{aligned}
& \max _{\beta_{i}^{t g t}} U\left(\beta_{i}^{t g t}\right) \\
& \text { s.t. } L \preccurlyeq L^{t g t} E,
\end{aligned}
$$

where $U$ is some utilization function representing the chosen resource management policy and the constraint simply states that the maximum eigenvalue of $L$ must be less than the scalar parameter $L^{t g t}$, i.e., $\bar{\lambda}(L) \leq L^{t g t}<1$. The RRM algorithm based on the above optimization problem will be referred to as the optimal algorithm. Solving this optimization problem requires complete knowledge of the matrix $\left[z_{i, j}\right]$ in the central node. A centralized solution like this therefore comes with the price of heavy signaling and/or slow adaptation to the changes in radio environment the users experience. In practice this leads to a demand for back-off in the optimization, which yields decreased utilization.

\section{B. A Semi-Centralized Algorithm}

The fundamental idea is to limit the intercell interference, $I_{k, j}, k \neq j$. The variable $L_{k, j}$ is a measure of how much load cell $k$ introduces in cell $j$. More specifically $L_{k, j} I_{k}^{t o t}$ is the intercell interference power users in cell $k$ introduce in cell $j$.

One way of limiting the off diagonal elements of $L$, i.e., to limit the intercell interference, is to limit each term in (2). A natural strategy is then that users with small relative power gain $z_{i, j}$ can be given a higher $\beta_{i}^{t g t}$. Feeding back complete knowledge on the relative power gains from the base stations to a central node requires heavy signaling, and is thus avoided in practice if possible. The amount of information sent from each base station to the central node can be reduced if, instead of sending all users' relative power gain measurements, only the average is sent, i.e., the vector $\operatorname{mean}_{i \in c_{k}} z_{i, j}$ is sent from each base station. This way, the amount of information sent to the central node depends only on the number of base stations and not on the number of users. Using the information on average relative power gain values, a matrix $Y=\left[Y_{k, j}\right]$ can be compiled,

$$
Y_{k, j} \triangleq \frac{1}{M_{k}} \sum_{i \in c_{k}} z_{i, j}
$$

\footnotetext{
${ }^{2}$ The notation $A \prec B$ means that the maximum eigenvalue of $A-B$ is less than 0 , while $x<y$ where $x$ and $y$ may be vectors, means component wise inequalities.
} 
where $M_{k}$ is the number of users in cell $k$. Introduce the matrix $\bar{L}=\left[\bar{L}_{k, j}\right]$ as

$$
\bar{L}_{k, j} \triangleq s_{k} Y_{k, j}
$$

where $s_{k}$ is a scalar.

Lemma 2: If $L_{k, j} \leq \bar{L}_{k, j} \forall k, j \in\{1, B\}$ and $\bar{\lambda}(\bar{L}) \leq$ $L^{t g t}$ then $\bar{\lambda}(L) \leq L^{t g t}$.

Proof: Follows from the fact that increasing an element of a positive matrix can not decrease the maximum eigenvalue.

If the resource assignment algorithm chooses target CTIR values such that

$$
L_{k, j}=\sum_{i \in c_{k}} \beta_{i}^{t g t} z_{i, j} \leq \bar{L}_{k, j} \forall k, j \in\{1, B\},
$$

then, according to lemma $2, \bar{\lambda}(\bar{L}) \leq L^{\text {tgt }}$ yields $\bar{\lambda}(L) \leq$ $L^{t g t}$. An interpretation of $s_{k}$ as a resource pool is given by studying Equation (5) in the case when $k=j$, i.e., on the diagonal of $L$ (note that $z_{i, K_{i}}=1 \forall i$ ),

$$
L_{k, k}=\sum_{i \in c_{k}} \beta_{i}^{t g t} z_{i, k}=\sum_{i \in c_{k}} \beta_{i}^{t g t} \leq \bar{L}_{k, k}=s_{k},
$$

i.e., $s_{k}$ is an upper bound on the sum of the target CTIR values of users connected to base station $k$.

Lemma 3: If

$$
\left(\begin{array}{cc}
E & \bar{L} \\
\bar{L}^{T} & L^{t g t^{2}} E
\end{array}\right) \succeq 0
$$

then $\bar{\lambda}(\bar{L}) \leq L^{t g t}$.

Proof: See the appendix.

Theorem 2 (System Feasibility): A system using a resource allocation algorithm that meets the inequalities in Equations (5) and (6) is feasible if $L^{t g t}<1$.

Proof: As the requirement in Equation (6) is met, $\bar{\lambda}(\bar{L}) \leq L^{t g t}$. According to Lemma $2, \bar{\lambda}(L)$ is then also less than $L^{t g t}$ if the inequality in (5) is met. Finally, according to Theorem 1 this yields feasibility if $L^{t g t}$ is less than 1 .

\section{The Algorithm}

We propose an algorithm choosing the users' target CTIR values as the solution to optimization problems in each base station. In order to meet the requirements of Theorem 2, however, a central node is required.

The resource pools, $s_{k}$, given to the base stations is produced by solving an optimization problem in the central node as well. In order to guarantee system feasibility the inequality in (6) has to be met. There may also be constraints on the users' possible $\beta_{i}^{t g t}$ assignments given by $\beta_{\text {min }}$ and $\beta_{\max }$. The optimization problem in the central node is thus

$$
\begin{aligned}
& \max _{s} U(s) \\
& \text { s.t. }\left\{\begin{array}{l}
\left(\begin{array}{cc}
E & \bar{L} \\
\bar{L}^{T} & L^{t g t^{2}} E
\end{array}\right) \succeq 0, \\
\bar{L}_{k, j}=s_{k} Y_{k, j}, k, j=1,2, \ldots, B . \\
s_{k}=\sum_{i \in c_{k}} \beta_{i}^{t g t} \\
\beta_{\text {min }} \leq \beta_{i}^{t g t} \leq \beta_{\max }, i=1,2, \ldots, M .
\end{array}\right.
\end{aligned}
$$

As utility function, $U(s)$, one should choose a convex function as this makes (7) a convex problem [15]. A possible choice is a greedy version

$$
U_{g}(s) \triangleq \sum_{k=1}^{B} s_{k},
$$

which simply maximizes the system throughput, or one may choose

$$
U_{f}(s) \triangleq \sum_{k=1}^{B} M_{k} s_{k},
$$

which is more fair to users in crowded cells. Obviously other choices of utility function can be made in the local base stations. We have only used $U_{g}(s)$ above.

To guarantee system feasibility, the inequality in Equation (5) must also be met. In this algorithm, this is done by the choice of target CTIR values, $\beta_{i}^{t g t}$, in the base stations. Since the actual resource assignments is done in the base stations the decisions can be based on local information and with a much higher update rate than what the central node operates on. The local optimization problem in base station $k$ is the linear program,

$$
\begin{aligned}
& \max _{\beta_{i}^{t g t} \in c_{k}} \sum_{i \in c_{k}} \beta_{i}^{t g t} \\
& \text { s.t. }\left\{\begin{array}{l}
\sum_{i \in c_{k}} \beta_{i}^{t g t} z_{i, j} \leq Y_{k, j} s_{k} \forall j \\
\beta_{\text {min }} \leq \beta_{i}^{t g t} \leq \beta_{\max }, \forall i \in c_{k} .
\end{array}\right.
\end{aligned}
$$

The requirement that the combined target CTIR values must be less than or equal to $s_{k}$ in base station $k$, is handled by the first constraint when $j=k$. If the first constraint is respected in all base stations, each element of $L$ is indeed upper bounded by the corresponding element in $\bar{L}$, i.e., Equation (5) is met.

As the maximum eigenvalue is less than or equal to the maximum singular value of any matrix [16], the matrix inequality in (7) implies a more strict requirement ${ }^{3}$. Put another way, the maximum eigenvalue $\bar{\lambda}(\bar{L})$ may be less than $L^{t g t}$ even if the matrix constraint is met with equality. This implies a loss in utilization. By scaling all users' $\beta^{t g t}$ values with $L^{t g t} \bar{\lambda}(\bar{L})^{-1}$, while respecting the upper bound on allowed $\beta^{\text {tgt }}$ values in (7), this loss can be partially regained.

The RRM algorithm using the optimization problems (7) and (10) will be referred to as the semi-centralized algorithm.

1) Algorithm Summary: To summarize, the algorithm uses two optimizations, one in the central node and one in each base station. Problem (7) is solved in the central node. To define the matrix $\bar{L}$ used therein it needs the matrix $Y$ which is compiled by information received from the local nodes, see Figure 1. The actual resource assignment is done in the base stations which have more detailed and up to date information on the situation in the cells. The target CTIR values $\left(\beta_{i}^{t g t}\right)$ are chosen as the solution to problem (10).

\footnotetext{
${ }^{3}$ The optimization results in a maximum singular value less than $\bar{L}$.
} 
2) Properties of the Algorithm: Since system feasibility is guaranteed by the decisions made in the central node, the base stations can focus on improving system performance by using information only locally available. Examples of manners in which this can be done are

- Fast adaptation to varying radio environment (such as multi path fading)

- Fast adaptation to transmission requests (buffer sizes)

- Base stations can, on their own, adapt to and take advantage of user movement

- Limited user transmission power can be respected in the local node by recursively reassigning the target CTIR values.

The purpose of feeding back $Y_{k, j}$ is that the central node then has an idea of how much the different cells interfere with each other. If, for example, many users in cell $k$ are close to the boarder between cell $k$ and cell $j, Y_{k, j}$ will be relatively large and the central node can then choose $s_{k}$ smaller in favor of other base stations' resource assignments, $s_{j}, j \neq k$.

As the users move around in the cell or even enter other cells, the local algorithms adapt the target CTIR values so that the constraint in (10) is always met. This constraint is crucial for system feasibility.

All optimization problems of this algorithm, the one in the central node as well as those in the local nodes, are of standard types. As such, there are efficient applicable to them.

\section{A Decentralized Algorithm}

As comparison, we will use an algorithm simply looking at the number of users in the own cell. A truly decentralized solution will be used. As there is no feedback used in this case, the central node has to be very conservative when setting the size of the resource pools $\left(s_{k}\right)$ given to the base stations. Even so, there is no guarantee that the system will be feasible.

The resource pool will be chosen as

$$
s_{k}=s_{0}, k=1,2, \ldots, B,
$$

where $s_{0}$ is a fixed parameter. This parameter can be seen as a tuning parameter and will be chosen beforehand. While a higher $s_{0}$ enables better utilization, it also increases the probability of too much uplink interference, or even infeasibility. The users in base station $k$ will get

$$
\beta_{i}^{t g t}=\frac{s_{0}}{M_{k}} .
$$

This RRM algorithm will be referred to as the decentralized algorithm.

\section{Simulations}

We have run a set of simulations. The simulation scenario is a set of $B=9$ cells distributed over 3 sites. A wrap around technique is used to eliminate boarder effects.

The offered load (amount of users) is constant during one simulation. Monte Carlo simulations are used for increased accuracy.
After one of the RRM algorithm explained in Section IV has assigned $\beta_{i}^{t g t}$ values to all users, the values are corrected to satisfy a transmission power constraint of $P^{\max }=0.2 \mathrm{~W}$.

Furthermore, $10 \%$ of all users are not allowed to connect to one of the nine cells. This models a user passing through a pico-cell without making a hand over to it.

The same base station positions are used in all simulations but the users are spread randomly before each simulation.

Table II shows parameters used in the simulations. The

TABLE II

SIMULATION PARAMETERS

\begin{tabular}{|c|c|}
\hline$B$ & 9 \\
\hline Max transmission power & $0.2 \mathrm{~W}$ \\
\hline Average Cell Radius & $750 \mathrm{~m}$ \\
\hline$\beta_{\min }$ & 0 \\
\hline$\beta_{\max }$ & 1 \\
\hline No. of MC simulations & 40 \\
\hline
\end{tabular}

a)
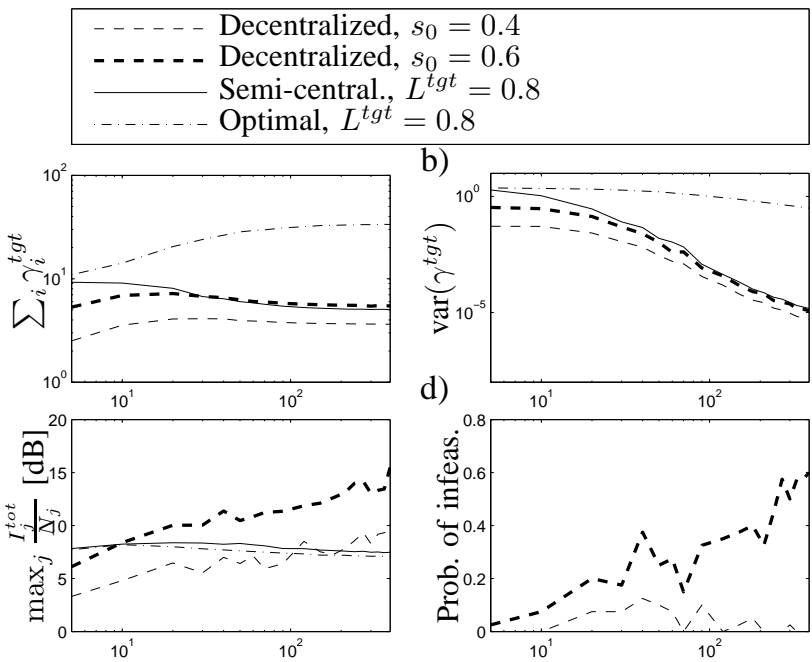

Fig. 2. Comparison of the RRM algorithms introduced in Section IV.It is the total number of available users $(M)$ on all x-axes. The bottom right graph shows the relative frequency of infeasible assignments.

main result is shown in graph $d$ of Figure 2. As opposed to the semi-centralized algorithm, the decentralized algorithm occasionally assigns resources such that the system becomes infeasible, especially when the more aggressive choice of $s_{0}=0.6$ is used.

Using $s_{0}=0.6$ (the thick dashed line) in the decentralized algorithm yields approximately the same resource utilization as the semi-centralized algorithm (graph a), only the decentralized algorithm causes much higher maximum noise rise, $\frac{I_{j}^{t o t}}{N}$ (graph c). The semi-centralized algorithm requires a maximum noise rise level of reasonable 6 to 7 $\mathrm{dB}$ while the decentralized algorithm causes a maximum noise rise of approximately $15 \mathrm{~dB}$. The power control is generally assumed to operate unsatisfactory when the noise rise is above 6 or $7 \mathrm{~dB}$.

There are two reasons why the semi-centralized algorithm provides a manageable noise rise level. One being that there 
is a guarantee that the noise rise level is limited since we have an upper bound on the actual uplink load, unlike the decentralized algorithm which simply limits the load caused by the own cell. However, it should be mentioned that the type of load limited by the semi-centralized algorithm is not directly related to the uplink noise rise [17]. A second reason why the uplink noise rise level is lower when using the semi-centralized algorithm is that the algorithm considers the relative power gain, instead of just the power gain to the own cell. This prevents the $10 \%$ of the users who are not connecting to the pico-cell to introduce too much interference in the pico-cell. Put another way, by limiting the intercell interference the algorithm manage to keep the noise rise lower while providing a higher throughput.

The decentralized and semi-centralized algorithms provide approximately equal variation in users' target CIR (graph c). This indicates that the semi-central algorithm does not prioritize users close to a base station. In fact, both algorithms try to give equal target CTIR to all users in the same cell. In the end, however, the target values may be changed due to limited transmission powers.

The difference between the semi-centralized algorithm and the optimal algorithm is that total target CIR (graph a) has been traded for decreased variance (graph b). The two algorithms cause approximately the same noise rise.

Finally, using $s_{0}=0.4$ instead of $s_{0}=0.6$ results in a slightly decreased sum of target CIR values, while the maximum noise rise level still reaches inappropriate levels.

\section{Conclusions}

We have proposed and evaluated a robust resource allocation algorithm. Robust in the sense that it always exists finite transmissions power to support the services given to the users. The proposed algorithm uses an amount of signaling between local and central nodes only dependent on the number of base stations.

Our solution is to define and solve two optimizations problems. One in the central node to guarantee system feasibility and one in the base stations to increase performance. Thanks to the local algorithms in the base stations the resource management can adapt to fast changes in the radio environment and users' service requests. As the constraints from the central node is given in as a resource pool, the base stations are given more freedom when allocating resources to the users.

Simulations show that the proposed algorithm is not only robust but manages to maintain the users' transmission powers on such a level that the power control algorithm operates satisfactory on. Furthermore, the proposed algorithm has no problems with handling a situation where some users may cause severe intercell interference in a scenario where there is a hierarchy of cells. This case is one which an algorithm only considering the impact on the own cell can not handle well.

\section{APPENDIX}

A. Proof of Lemma 3

The Schur Complement of a matrix is a useful tool for establishing whether a matrix is positive definite or not [18]. Consider the matrix

$$
X=\left(\begin{array}{cc}
A & B \\
B^{T} & C
\end{array}\right),
$$

where $A$ is a symmetric matrix. Assume that $\operatorname{det}(A) \neq 0$ and define the Schur complement as the matrix

$$
S=C-B^{T} A^{-1} B .
$$

If $A \succ 0$, the following two statements are equivalent

- $X \succeq 0$

- $S \succeq 0$.

Comparing $X$ with the extended matrix in the lemma (6), we get

$$
A=E, C=L^{t g t^{2}} E \text { and } B=\bar{L} \text {. }
$$

Now, since $A=E \succ 0$, the requirement in the lemma implies

$$
L^{t g t^{2}} E-\bar{L}^{T} \bar{L} \succeq 0 \Leftrightarrow \bar{L}^{T} \bar{L} \preceq L^{t g t^{2}} E .
$$

This means that

$$
\lambda_{k}\left(\bar{L}^{T} \bar{L}\right) \leq L^{t g t^{2}}, k=1,2, \ldots B .
$$

As the maximum eigenvalue of a matrix is less than or equal to its maximum singular value [16] this gives

$$
\bar{\lambda}(\bar{L}) \triangleq \max _{k} \lambda_{k}(\bar{L}) \leq \sqrt{\bar{\lambda}\left(\bar{L}^{T} \bar{L}\right)} \leq L^{t g t} .
$$

\section{REFERENCES}

[1] J. Zander, "Radio resource management - an overview," in Proc. IEEE Vehicular Technology Conference, Atlanta, GA, USA, April 1996.

[2] J. Herdtner and E. Chong, "Analysis of a class of distributed asynchronous power control algorithms for cellular wireless systems," IEEE Journal on Selected Areas in Communications, vol. 18, no. 3, Mar 2000.

[3] 3GPP, "FDD Enhanced Uplink; Overall description," 3GPP, Technical Specification 3GPP TS 25.309, 2005.

[4] S. Parkvall, J. Peisa, J. Torsner, and P. Malm, "WCDMA enhanced uplink - principles and basic operation," in Proc. IEEE Vehicular Technology Conference, Stockholm, Sweden, May 2005, to appear.

[5] X. Duan, Z. Niu, D. Huang, and D. Lee, "A dynamic power and rate joint allocation algorithm for mobile multimedia DS-CDMA networks based on utiliity functions," in Proc. IEEE Personal, Indoor and Mobile Radio Communications, Sep 2002.

[6] Y. Ishikawa and N. Umeda, "Capacity design and performance of call admission control in cellular CDMA systems," IEEE Journal on Selected Areas in Communications, vol. 15, no. 8, 1997.

[7] F. Gunnarsson, E. Geijer Lundin, G. Bark, and N. Wiberg, "Uplink admission control in WCDMA based on relative load estimates," in Proc. International Conference on Communications, New York, April 2002.

[8] V. Rodriguez, D. Goodman, and Z. Marantz, "Power and data rate assignment for maximal weighted throughput in 3G CDMA: a global solution with two classes of users," in Proc. IEEE Wireless Communications and Networking Conference, March 2004.

[9] A. Sampath, P. Sarath Kumar, and J. M. Holtzmann, "Power control and resource management for a multimedia CDMA wireless system," in Proceedings IEEE Personal, Indoor and Mobile Radio Communications, Toronto, Ont., Canada, Sep 1995. 
[10] T. Javidi, "Decentralized rate assignments in a multi-sector CDMA network," in Proc. IEEE Global Telecommunications Conference, Dec 2003.

[11] R. Rezaiifar and J. Holtzman, "Proof of convergence for the distributed optimal rate assignment algorithm," in Proc. IEEE Vehicular Technology Conference, Houston, TX, USA, May 1999.

[12] N. Feng, S.-C. Mau, and N. B. Mandayam, "Pricing and power control for joint network-centric and user-centric radio resource management," IEEE Transactions on Communications, vol. 52, no. 9, 2004.

[13] J. Zander, "Optimum global transmitter power control in cellular radio systems," in Personal, Indoor and Mobile Radio Communications., IEEE International Symposium on, September 1991.

[14] F. R. Gantmacher, The Theory of Matrices, Vol. II, Ch. XIII. New York, NY, USA: Chelsea Publishing Company, 1974.

[15] S. Boyd and L. Vandenberghe, Convex Optimization. The Edinburgh Building, Cambridge, CB2 2RU, UK: Cambridge University Press, 2004.

[16] G. H. Golub and C. van Loan, Matrix Computaions, third edition, $p$. 318. Baltimore, MD, USA: The Johns Hopkins University Press, 1996.

[17] E. Geijer Lundin, "Uplink load in CDMA cellular systems," Linköping, Sweden, Tech. Rep., Oct 2003, licentiate Thesis, Linköping University, Linköping, Sweden.

[18] K. Zhou, J. Doyle, and G. K., Robust and Optimal Control. Upper Saddle River, NJ, USA: Prentice-Hall, 1995. 